\title{
Adding muscle power exercises to a strength training program for people with patellofemoral pain: protocol of a randomized controlled trial
}

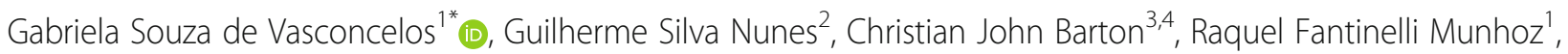
Maria Eduarda Chinotti Batista da Silva ${ }^{1}$, Giulia Keppe Pisani ${ }^{1}$, Bruna Calazans Luz ${ }^{1}$ and Fábio Viadanna Serrão ${ }^{1}$

\begin{abstract}
Background: Strong evidence supports the proximal combined with quadriceps strengthening for patellofemoral pain (PFP) rehabilitation. However, most reported rehabilitation programs do not follow specific exercise prescription recommendations or do not provide adequate details for replication in clinical practice. Furthermore, people with PFP have power deficits in hip and knee muscles and it remains unknown whether the addition of power exercises would result in superior or more consistent outcomes. Therefore, this study is designed to verify whether the benefits of a rehabilitation program addressing proximal and knee muscles comprising power and strength exercises are greater than those of a program consisting of strength exercises only.
\end{abstract}

Method: This study will be a randomized controlled trial that will be conducted at university facilities. A minimum of 74 people with PFP between the ages of 18 and 45 years will be included. The experimental group will engage in a 12-week resistance training program focusing on proximal and knee muscles using power and strength exercises. The control group will engage in a 12-week resistance training program focusing on proximal and knee muscles using strength exercises only. Primary outcomes will be pain intensity and physical function; and secondary outcomes will be kinesiophobia, self-reported improvement, quality of life, peak hip and knee torque, and hip and knee rate of force development. The primary outcomes will be evaluated at baseline, and after 6 weeks, 12 weeks, 3 months, 6 months, and 1 year. The secondary outcomes will be evaluated at baseline and immediately after the interventions. Therapists and participants will not be blinded to group allocation.

Discussion: This randomized clinical trial will investigate if adding power exercises to a progressive resistance training may lead to more consistent outcomes for PFP rehabilitation. The study will provide additional knowledge to support rehabilitation programs for people with PFP.

Trial registration: ClinicalTrials.gov NCT 03985254. Registered on 26 August 2019.

Keywords: Knee, Muscle strength, Exercise therapy, Quality of life

\footnotetext{
* Correspondence: vasconcelosgs@gmail.com

'Physical Therapy Department, Federal University of Sao Carlos (UFSCar), Rodovia Washington Luis Km 235, São Carlos, São Paulo CEP 13565-905, Brazil

Full list of author information is available at the end of the article
}

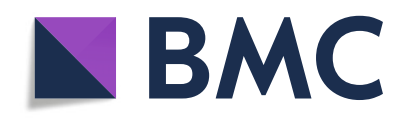

(- The Author(s). 2021 Open Access This article is licensed under a Creative Commons Attribution 4.0 International License, which permits use, sharing, adaptation, distribution and reproduction in any medium or format, as long as you give appropriate credit to the original author(s) and the source, provide a link to the Creative Commons licence, and indicate if changes were made. The images or other third party material in this article are included in the article's Creative Commons licence, unless indicated otherwise in a credit line to the material. If material is not included in the article's Creative Commons licence and your intended use is not permitted by statutory regulation or exceeds the permitted use, you will need to obtain permission directly from the copyright holder. To view a copy of this licence, visit http://creativecommons.org/licenses/by/4.0/ The Creative Commons Public Domain Dedication waiver (http://creativecommons.org/publicdomain/zero/1.0/) applies to the data made available in this article, unless otherwise stated in a credit line to the data. 


\section{Introduction}

Patellofemoral pain (PFP) is characterized by retropatellar and/or peripatellar pain that is aggravated during activities that increase patellofemoral joint loading (e.g., squatting and ascending/descending stairs) [1], with an annual prevalence in the general population of around $22.7 \%$ [2]. Although PFP was previously considered as a self-limiting condition, recent studies suggest that alterations and symptoms may persist for several years [3-5]. Chronic pain associated with PFP has a negative impact on an individual's level of physical activity and quality of life, interfering with work, activities of daily living, and physical exercise [5-7].

Previous studies reported that people with PFP have decreased strength in hip $[8,9]$ and knee muscles $[10$, 11]. Additionally, other parameters of hip and knee muscle capacity seem to be altered in people with PFP, such as rate of force development (RFD) and power [12-15]. Therefore, exercise therapy addressing these deficits may be important for PFP rehabilitation. Some studies reported the effects of rehabilitation programs for PFP patients focused on proximal muscles combined with quadriceps strengthening [16-23]. Two systematic reviews concluded that the combination of proximal and quadriceps rehabilitation results in greater benefits on pain intensity and physical function compared to isolated quadriceps rehabilitation $[24,25]$. Nowadays, the combination of hip and knee muscles exercises presents the best level of evidence for pain reduction and physical function improvement during PFP rehabilitation [26]. Despite improvements in pain and physical function, the effects of resistance training on other characteristics seen in people with PFP such as kinesiophobia, quality of life, and catastrophization are unclear [27-30].

According to the American College of Sports Medicine (ACSM), to achieve sustained improvements in muscle capacity, specific evidence-based guidelines must be followed, such as load progression, number of sets and repetitions, time to rest, weekly frequency, and duration of this training [31, 32]. In a systematic review conducted by Lack et al. [24], the included studies evaluated the effects of resistance training targeting proximal muscles of people with PFP. After further analysis using the ACSM criteria, the authors concluded that three out of 14 studies actually incorporated protocols which could evoke improvements in muscle strength $[18,21,22]$. However, none of the included studies completely accomplished the ACSM guidelines for structural strength gains (more than 8 weeks), as the protocols lasted $4[18,21]$ to 8 weeks [22]. Additionally, according to Lack et al. [24], only one study included power exercises [33], even though the exercise protocol was not clearly described for replication in clinical practice or research. This is a common issue among PFP randomized controlled trials highlighted in the systematic review by Holden et al. [34]. The systematic review included 38 studies and the authors concluded that exercise prescriptions are poorly described, which prevents them from being implemented in clinical practice.

Although hip and knee strength exercises present the best level of evidence for pain reduction and physical function improvement in people with PFP [24-26], little is known in relation to their long-term effects. To date, only the study by Fukuda et al. [21] investigated the long-term effects of exercise (12 months) and the study reported that hip and knee strength exercises result in greater benefits on pain intensity and physical function compared to quadriceps strengthening alone. However, rehabilitation programs with exercises that correct other muscle capacity deficits, such as muscle power, can result in superior or more persistent benefits than those with only strengthening exercises. More recently, Barton et al. [30] demonstrated that a 12-week progressive resistance training program (hip and knee strength and power exercises) is feasible and is associated with improvements in pain, physical function, and muscle capacity (strength and power) in people with PFP. Nonetheless, it is unknown whether the benefits of a proximal combined with quadriceps rehabilitation comprising power and strength exercises are greater than those of a proximal combined with quadriceps rehabilitation consisting of strength exercises only.

To improve the knowledge regarding the effects of muscle power exercises on PFP rehabilitation, the main aim of the study will be to verify if including power exercises to a strength training program addressing proximal and knee muscles provides better outcomes in relation to intensity pain and physical function, compared to a strength training program only, in the short, medium, and long term in people with PFP. Furthermore, the aim will be to verify whether the proximal combined with quadriceps rehabilitation comprising power and strength exercises will result in superior benefits on kinesiophobia, selfreported improvement, quality of life, and muscle capacity (increased peak hip abduction and extension torque, and peak knee extension torque; increased hip abduction and extension RFD, and knee extension RFD). The hypothesis of the study is that people with PFP undergoing the proximal combined with quadriceps rehabilitation comprising power and strength exercises will improve considerably concerning the evaluated outcomes when compared to people undergoing the proximal combined with quadriceps rehabilitation composed by strength exercises only. 


\section{Methods}

\section{Trial design}

This is a randomized controlled trial. The study will be developed in six stages, according to Fig. 1. The baseline assessment will consist of evaluating anthropometric and demographic data, in addition to pain intensity, physical function, kinesiophobia, quality of life, and muscle capacity. After 1 week, the intervention phase will be initiated, which will consist of two different intervention protocols for 12 weeks, according to the group allocation. Six weeks after starting the intervention, the intensity of pain, physical function, kinesiophobia, and quality of life will be assessed. At the end of the intervention protocols, the participants will be evaluated for pain intensity, physical function, kinesiophobia, quality of life, muscle capacity, and self-reported improvement. After 3 months, 6 months, and 1 year, participants will be re-evaluated for pain intensity and physical function through online questionnaires.
The lower limb of the affected side will be assessed. In cases of bilateral symptoms, the more painful lower limb will be assessed [12].

The study will be conducted at the Laboratory of Evaluation and Intervention in Orthopedics and Traumatology (LAIOT) at the Federal University of São Carlos (UFSCar), São Carlos, São Paulo, Brazil, and will follow the CONSORT guideline [35] and the TIDieR checklist to describe the interventions [36]. The present protocol was reported according to the SPIRIT guideline [37].

\section{Ethics}

The research was approved by the University Research Ethics Committee (CAAE: 12417019.8.0000.5504). Each participant will receive explanations regarding research objectives, anonymity of their data, and freedom to participate. Participants will sign an Informed Consent Term (ICT). This study will respect the ethical precepts

\begin{tabular}{|c|c|c|c|c|c|c|c|c|}
\hline \multirow{3}{*}{ TIMEPOINT } & \multicolumn{8}{|c|}{ STUDY PERIOD } \\
\hline & \multirow{2}{*}{ 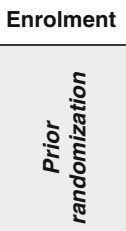 } & \multirow{2}{*}{$\begin{array}{c}\text { Allocation } \\
0 \\
0\end{array}$} & \multicolumn{5}{|c|}{ Post allocation (12-week intervention) } & \multirow{2}{*}{ 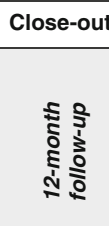 } \\
\hline & & & 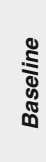 & 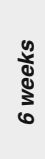 & 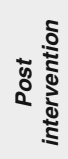 & 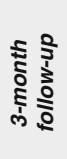 & 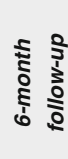 & \\
\hline \multicolumn{9}{|l|}{ ENROLMENT: } \\
\hline Eligibility screen & $x$ & & & & & & & \\
\hline Informed consent & $x$ & & & & & & & \\
\hline Allocation & & $x$ & & & & & & \\
\hline \multicolumn{9}{|l|}{ INTERVENTIONS: } \\
\hline \multicolumn{9}{|l|}{ Strength Training } \\
\hline \multicolumn{9}{|l|}{$\begin{array}{c}\text { Strength and } \\
\text { Power Training }\end{array}$} \\
\hline \multicolumn{9}{|l|}{ ASSESSMENTS: } \\
\hline Pain Intensity & & & $X$ & $\mathrm{X}$ & $\mathrm{X}$ & $\mathrm{X}$ & $\mathrm{X}$ & $\mathrm{X}$ \\
\hline Physical Function & & & $\mathrm{X}$ & $\mathrm{X}$ & $\mathrm{X}$ & $\mathrm{X}$ & $\mathrm{X}$ & $\mathrm{X}$ \\
\hline Kinesiophobia & & & $\mathrm{X}$ & & $\mathrm{X}$ & & & \\
\hline Quality of Life & & & $\mathrm{X}$ & & $\mathrm{X}$ & & & \\
\hline $\begin{array}{c}\text { Isometric Peak } \\
\text { Torque }\end{array}$ & & & $\mathrm{X}$ & & $\mathrm{X}$ & & & \\
\hline $\begin{array}{l}\text { Rate of Force } \\
\text { Development }\end{array}$ & & & $\mathrm{X}$ & & $\mathrm{X}$ & & & \\
\hline $\begin{array}{l}\text { Self-improved } \\
\text { Perception }\end{array}$ & & & & & $\mathrm{X}$ & & & \\
\hline
\end{tabular}

Fig. 1 Participant timeline: schedule of enrolment, interventions, and assessments 
of Resolution CNS 510/2016 and be performed according to the Declaration of Helsinki. It was registered in ClinicalTrials.gov, on 26 August 2019, under number ID: NCT 03985254.

\section{Participants and setting}

Potential participants will be recruited from the community, gyms, or social media, and a minimum of 74 participants will be included in the study. A researcher will perform a preliminary screening of the eligibility criteria and will explain the study procedures during a telephone call. If the potential participants likely fulfill the inclusion criteria and declare their agreement to participate, the diagnosis of PFP will be confirmed by a physiotherapist through a physical examination [38]. This physiotherapist $(\mathrm{BCL})$ will explain the study procedures and will obtain written consent from patients willing to participate in the trial.

To be included, the participants should meet the following inclusion criteria: (i) men and women affected by PFP (unilateral or bilateral) aged between 18 and 45 years; (ii) insidious onset of symptoms unrelated to a traumatic event; (iii) presenting retropatellar or peripatellar pain (3/10 points according to the visual analog scale-VAS) in at least two of the following functional activities: stair negotiation, running, kneeling, squatting, sitting for long periods, or jumping; and (iv) presence of pain for at least 2 months [17]. The exclusion criteria will be history of surgery to the knees; history of injury or pain in the hip; patellar instability; pain on palpation of the patellar tendon, iliotibial band, Hoffa fat, pes anserinus tendons or knee joint line; signs or symptoms of meniscal or ligamentous knee injuries; presence of Osgood-Schlatter or Sinding-Larsen-Johansson syndrome; and any vestibular, neurological, or musculoskeletal alterations that interfere with or contraindicate the measurement procedures of this study [22, 39]. After randomization, the criterion for dropping out will be the participants not attending the assessments (6 weeks, post intervention and follow-ups).

\section{Sample size}

The sample size of this study was calculated considering a statistical power of $80 \%$, alpha of $5 \%$, and an estimated $15 \%$ of sample losses. In order to detect a 2-point difference in pain NRS [40] with a standard deviation of 2.8 points [41], a sample size calculation indicated 37 participants in each group.

\section{Randomization and allocation}

All participants who give consent for participation and who fulfill the inclusion criteria will be randomized to the Strength Training Group (STG) or to the Power and Strength Training Group (PSTG) with a 1:1 allocation ratio. Randomization will be performed using consecutively numbered, sealed and opaque envelopes previously prepared and randomly assigned by a random number generator program (www.randomization.com). Allocation concealment will be ensured, since the randomization code will only be released after the participant has been included in the clinical trial. A researcher not involved in the assessment process will perform the randomization and participant allocation to the groups by opening the envelopes after the baseline evaluation. The physiotherapist, responsible for the intervention, will open the envelope and, will find the treatment condition to be conducted in this participant. The researcher responsible for assessments is not allowed to receive information about the group allocation. Thus, randomization will be conducted without any influence of the physiotherapists and researchers responsible for assessments and interventions.

\section{Interventions}

Participants from both groups will perform the supervised training program three times per week for 12 weeks, with at least $24 \mathrm{~h}$ of interval between intervention sessions. No instruction for home exercise will be given. Participants will be instructed to maintain their physical and sports activities.

The duration of each session for the STG will be around $60 \mathrm{~min}$ and for the SPTG around $75 \mathrm{~min}$. All sessions will be supervised by an experienced physiotherapist ( $>5$ years). The description of the exercises has been made simple and clear; therefore, physiotherapists will need minimal training to apply the exercises (Additional file 1 - supplementary material).

The STG and SPTG programs will be based on the training principles recommended by the American College of Sport Medicine (ACSM) [32]. The exercises were chosen based on a study that successfully applied the protocol to people with PFP [30] and other strength training studies [22, 42-45].

To perform the exercises, ankle weights, free weights and/or dumbbells, and elastic bands will be used. The load to be used in the exercises of both groups will be determined based on the 10RM (repetition maximum) test to estimate the 1RM-the 10RM load indicates approximately $75 \%$ of the $1 \mathrm{RM}$ load [46]. As the participants have PFP, the 1RM test could exacerbate the symptoms. For exercises using an elastic band, the 1RM test will be performed. The 1RM will be considered the highest elastic band (considering the color of the elastic), in which the participant can perform a single repetition [22].

Regarding pain management during exercise, a level equal to or lower than three points in VAS will be accepted, and if any participant presents pain above this level, the exercise will be modified according to the variations that are also in the exercise protocol 
(Additional file 1). In addition to pain management, the presence of muscle failure will also be monitored. Exercise will be interrupted if muscle failure occurs, which will be considered as the participant's inability to move a specific load beyond a critical joint angle [47] or as the inability to complete a repetition in the stipulated range of motion due to fatigue [48].

As an adherence strategy to encourage participants attending training sessions, both groups will receive face-to-face education sessions about possible causes and consequences of PFP, pain management, importance of physical activity for the treatment, and other questions that may arise during the intervention period. In addition, telephone calls will be made to remind participants about the sessions. The intention is that the participants feel part of the treatment, understand how the intervention can help them, and, thus, attend the intervention sessions [49].

\section{Strength Training Group (STG)}

The strength training program will consist of applying resistance and progressive exercises for strength gain. Initially, the goal will be to develop neuromotor control and endurance (load $<50 \% 1 \mathrm{RM}$ ), and in subsequent weeks, the goal will be to develop muscle strength (load $>70 \%$ 1RM) (Fig. 2). The protocol will initially focus on hip and trunk muscles, and after 4 weeks of training, knee muscle exercises (weight-bearing or non-weightbearing) will be included.

At each training session, at least five exercises (out of a total of 15 exercises) will be chosen and applied by the therapist. From these five exercises, one will be for hip extensors, one for hip abductors, one for knee extensors, and two for the trunk. Additional file 1 provides a complete description of all exercises that may be applied to participants. According to the evolution of the participants, regarding pain and ease of execution, the therapist will perform the progression of the loads and even the substitution of one exercise for a more complex one as long as it is one of the program exercises.

\section{Power and Strength Training Group (PSTG)}

Participants assigned to this group will perform the same exercise program as the STG and power training exercises (fourth column of Additional file 1). Initially, the focus will be on neuromotor control and resistance development (load $<50 \% 1 \mathrm{RM}$ ). Afterwards, the goal will be to develop strength (load $>70 \% 1 \mathrm{RM}$ ) and muscle power (load between 40 and 60\% 1RM) (Fig. 3).

\section{Outcome measures}

Primary outcomes will be pain intensity and physical function, and secondary outcomes will be kinesiophobia, self-reported improvement, quality of life, peak hip and knee torque, and hip and knee rate of force development. The primary outcomes will be evaluated at baseline, and after 6 weeks, 12 weeks, 3 months, 6 months, and 1 year. The secondary outcomes will be evaluated at baseline and immediately after the interventions.

\section{Pain intensity}

Pain intensity will be measured using the Numeric Rating Scale (NRS-11), where 0 represents "no pain" and 10 represents "the worst pain possible." Participants will indicate their usual pain and worst pain during the past week [40].

\section{Physical function}

The Anterior Knee Pain Scale (AKPS), translated and validated into Portuguese [50], will be used to assess subjective symptoms such as anterior knee pain and functional limitations related to PFP. The items evaluated in the questionnaire are patellar subluxation, limp, pain, walking, climbing stairs, and sitting for a long time with bent knees. It has a score from 0 to 100 points, where 100 means no pain and/or functional limitations and 0 means constant pain and various functional limitations [51]. The AKPS is a reliable and valid instrument for assessing function in individuals with PFP [40].

\section{Kinesiophobia}

The evaluation of kinesiophobia will be performed by the translated and validated Portuguese version of the

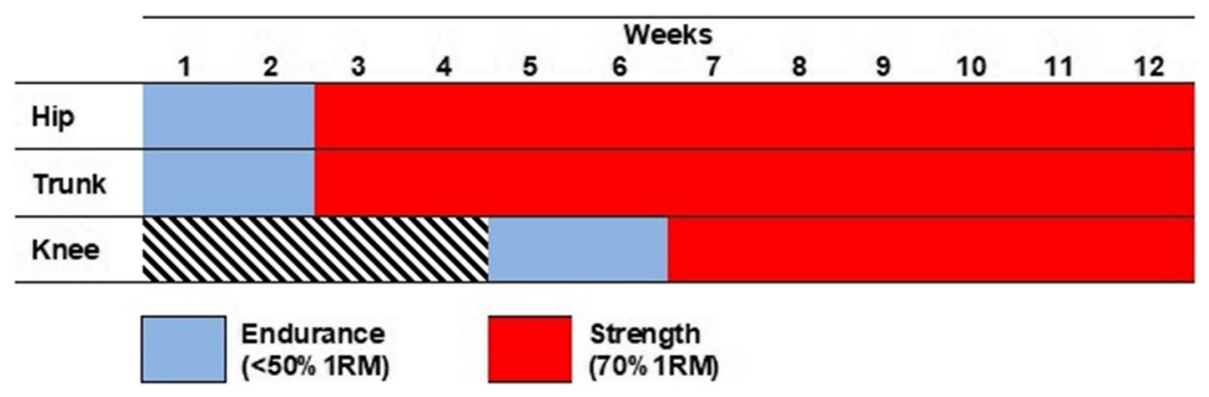

Fig. 2 Strength Training Group (STG) schedule 


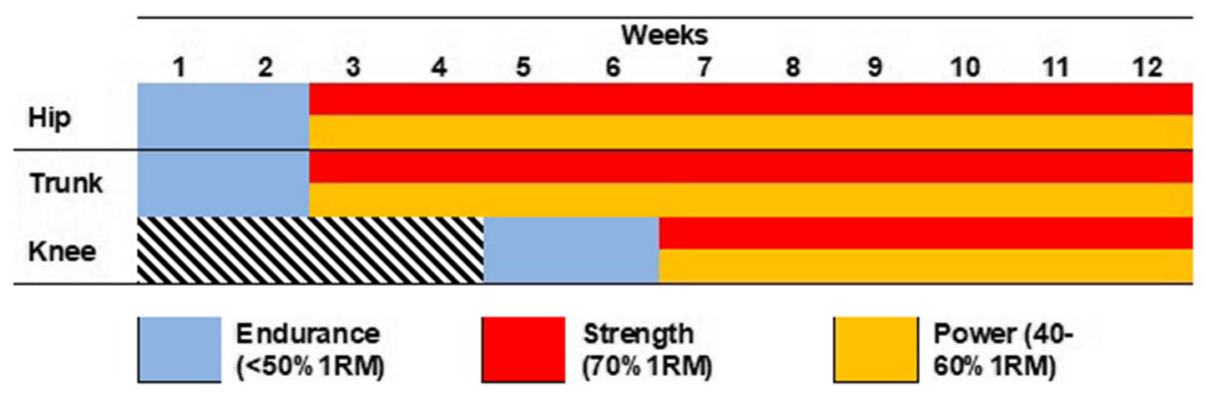

Fig. 3 Power and Strength Training Group (PSTG) schedule

Tampa Scale for the Kinesiophobia questionnaire [52], which consists of 17 items that assess fear of movement, injury or recurrence of injury [53]. This questionnaire is a four-point Likert scale, where the sum of the answers can vary from 17 to 68 , and scores higher than 37 indicate the presence of kinesiophobia [54].

\section{Quality of Life}

Participants will be assessed using the specific subscale for quality-of-life assessment of Knee Injury and Osteoarthritis Outcome Score (KOOS). This subscale comprises four questions and each one will be scored from 0 to 4 , where 0 represents extreme knee problems and 4 that there are no knee problems. The sum of these questions will be used for further analysis $[55,56]$.

\section{Muscle capacity}

Isometric torque and RFD of knee extensors, hip extensors, and abductors will be evaluated using an isokinetic dynamometer (Biodex Multi Joint System 3, Biodex Medical Systems Inc., New York, USA), with a sampling frequency of $100 \mathrm{~Hz}$.

- Knee extensors: the participants will be placed in a sitting position with hips at $85^{\circ}$ of flexion and neutral position for transversal and frontal planes, and the knee of the assessed limb at $60^{\circ}$ of flexion [13]. The resistance pad will be fixed with a velcro strip just above the lateral malleolus and the rotation axis of the dynamometer will be aligned with the lateral femoral epicondyle.

- Hip extensors: the participants will be in a prone position on the testing table of the dynamometer and their legs will be off the table. The assessed lower limb will be placed with the hip at $30^{\circ}$ of flexion, and the participants should keep the knee at $90^{\circ}$ of flexion and avoid hip rotations during the test. The resistance pad will be fixed with a velcro strip just above the popliteal fossa, and the rotation axis of the dynamometer will be aligned with the center of the hip joint in the sagittal plane near the greater trochanter of the femur $[12,57]$.

- Hip abductors: the participants will be placed in a side-lying position, whereby the top leg will be the assessed side. The assessed hip will be in neutral position for all three planes, the knee will also be in neutral position, and the participants will be instructed to keep their toes pointed forward and not to bend their knee during the test [58]. The resistance pad will be fixed with a velcro strip $5 \mathrm{~cm}$ above the upper edge of the patella and the rotation axis of the dynamometer will be aligned to a point representing the intersection of two lines: one line directed inferiorly from the posterior superior iliac spine towards the knee, and the other line oriented medially and posteriorly to the greater trochanter of the femur toward the midline of the body.

For the whole assessment, the participants should perform the contraction as powerfully and as quickly as possible and maintain the contraction for $5 \mathrm{~s}$. For each assessed muscle group, three maximal contractions will be collected with a 1-min interval between them. The participants will be verbally encouraged to achieve maximum power throughout all the contractions. Previously to the data collection, the participants will be familiarized with the tests by performing two submaximal contractions and one maximal contraction. A 3-min interval will be adopted between familiarization and data collection. Isometric torque measures will be normalized by body mass $(\mathrm{Nm} / \mathrm{kg} \times 100)$, and the highest value of the three repetitions will be used for statistical analysis for each muscle group [12].

A test-retest reliability study will be conducted to verify intra-examiner reliability of measurements by evaluating 10 participants in two moments, with an interval of 3 to 7 days.

To calculate the RFD, the highest value of the three repetitions for each muscle group will be used [12]. The normalized torque data will be exported and processed in the Matlab Software (Mathworks, Natick, Massachusetts, 
USA, version 2008b). RFD will be calculated using the slope of the torque/time curve. The slope will be obtained by dividing the variation of the normalized torque $(\mathrm{Nm} /$ $\mathrm{Kg} X 100$, represented as \%) by the time variation (ms) from the start of the contraction until $30 \%$ and $90 \%$ of the maximal isometric torque $[59,60]$. The beginning of the contraction will be defined as the moment when the isometric torque exceeds $2 \%$ of the peak torque [61]. Thus, higher RFD values indicate an increased ability to generate force rapidly [62].

\section{Self-reported improvement}

The self-reported improvement with reference to the start of the study will be quantified using the Global Rating of Change (GROC). This tool is a 15-point Likerttype scale that measures a patient's perception of a change in knee pain following a specific treatment [63]. The scale ranges from -7 (a very great deal worse) to + 7 (a very great deal better), with 0 (zero) indicating no change. Changes of 4 points or more on this scale have been previously considered as clinically important in patients with knee pain [64].

\section{Blinding}

The researcher in charge of the evaluations and the data processing will be blinded to the group allocation. The participants and the therapist will not be blinded to the group allocation due to the differences between our interventions which are easily detectable.

\section{Minimizing missing data}

In order to avoid and minimize missing data, as an adherence strategy to encourage participants to attend training sessions, both groups will receive face-to-face education sessions about possible causes and consequences of PFP, pain management, importance of physical activity for the treatment, and other questions that may arise during the intervention period. In addition, telephone contacts will be made to remind participants about the sessions and the evaluations. Regarding the 3 month-, 6 month-, and 1-year follow-ups, the researcher will contact the participant by telephone and send them the online questionnaire.

\section{Data management}

Data are being collected at the Laboratory of Evaluation and Intervention in Orthopedics and Traumatology (LAIOT) at the Federal University of São Carlos (UFSCar). Data will be stored at the Physical Therapy Department at UFSCar with a password-protected computer file, to which only researchers will have access. The main researcher will have a backup copy of all the information.
The study results will be released in conferences, such as scientific conferences, internationally and nationally, and through articles published in peer-reviewed journals. This research is a part of a $\mathrm{PhD}$ thesis, and the publishing rights are owned by the authors. The results will be frequently presented to the supervising professor (FVS).

\section{Data monitoring}

Researchers will monitor participants throughout the study development (assessment and interventions). The supervising professor (FVS), who will be blind to the group allocations, will monitor any adverse effects and perform database management and statistical analyses.

Adverse events during the study procedures, either during assessments, interventions, or follow-up, will be registered and reported. Adverse events will be considered any symptom or disease that is related or not to the evaluations and the intervention.

The University Graduate Program will supervise the integrity of the data, and the responsible Internal Data Monitoring Committee will have access to the patient allocation, while the whole analysis will be confidential. The supervising professor (FVS) will ensure that the University Graduate Program and Internal Data Monitoring Committee will be provided with access to source data/documents, ensuring the confidentiality of the participants.

\section{Harm}

Participant data will be carefully accessed, and all harm and complications of the treatment will be reported together with the other results of this trial, if any.

\section{Auditing}

The University Graduate Program will supervise the integrity of the data. Moreover, the results will be frequently presented to the supervising professor (FVS), who will be blind to the group allocations, and will verify if data are accurate, complete, and verifiable and that the conduct of the study complies with the currently approved protocol.

\section{Statistical analysis}

The intention-to-treat approach will be applied for all analyses [65]. The differences from baseline will be used in the analysis. The effects of intervention on the outcome measures will be assessed by analysis of variance (ANOVA). For pain and physical function (primary outcomes), a 2-by-6 analysis of variance will be used with the groups (STG and PSTG) as the independent factor and time (baseline, 6-week, post intervention, 3-month follow-up, 6-month follow-up, and 12-month follow-up) as the repeated factor. For kinesiophobia (secondary outcome), a 2-by-3 analysis of variance (2 groups and 3 
time points) will be used. For quality of life and muscle capacity (hip and knee torque; hip and knee RFD) (secondary outcomes), a 2-by-2 analysis of variance (2 groups and 2 time points) will be used. For GROC outcome (secondary outcomes), the reference criterion of treatment success will be a score of +6 (much improved) or higher and these data will be presented as a percentage. Moreover, chi-square tests will be performed to compare the percentage of patients who perceived much improvement in each group based on the GROC scale. Separate models will be used for each outcome measure. For significant group-by-time interactions, planned pairwise comparisons with post hoc Bonferroni will be used to determine differences. The mean difference and 95\% CI will be also calculated for each comparison. In cases of missing data, the multiple imputation method will be adopted to impute missing values [66], and per-protocol analysis will be also performed. The significance level will be 0.05 . All the statistical analysis will be performed using a Statistical Package for the Social Sciences software program (version 20.0, SPSS, Inc., Chicago, IL, USA), and the researcher who will perform the analysis will be blinded to the group allocation. Interim analyses will not be performed. No additional analyses will be performed in the trial.

\section{Discussion}

Knee pain is the second most prevalent condition, in which PFP is considered one of the most common forms of knee pain [67]. People with PFP have decreased hip $[8,9]$ and knee muscle strength $[10,11]$, and, as a result, many studies have focused on improving these deficits [16-23]. According to the latest PFP consensus [26], the proximal combined with quadriceps strengthening is the physiotherapeutic procedure that has the best evidence for pain reduction and function improvement in PFP patients.

Moreover, people with PFP present other proximal and knee muscle capacity deficits, such as rate of force development (RFD) and power [12-15]. Nunes et al. [12, 13] reported lower hip abductor and extensor and knee extensor rate of force development (RFD) during maximal isometric contraction in women with PFP compared to women without PFP. Similarly, according to the study by Ferreira et al. [15], women with PFP have lower hip abductor and knee extensor RFD during isometric, concentric, and eccentric contractions compared to women without PFP. Finally, using a linear position transducer, it was found that people with PFP had deficits of $31 \%$ and $29 \%$ in hip abductor and extensor power, respectively [14].

Although these recent studies have shown that patients with PFP have hip and knee muscle power deficits
[12-15], little is known in relation to power training effects in these people. Until the present moment, only two studies included power exercises for the treatment of these patients [30, 33]. Nevertheless, Tyler et al. [33] did not adequately describe which power exercises were applied, and Barton et al. [30] did not have a comparator group (isolated muscle strength protocol) to clarify whether adding power exercises to a strength training protocol results in greater benefits than muscle strength training alone.

In addition to these problems, others are identified in previously published studies with interventions for PFP. For example, the vast majority of them did not follow ACSM recommendations aiming to gain muscle strength (load progression, number of sets and repetitions, time to rest, weekly frequency and duration of this training) [24]. Another problem, presented in the systematic review by Holden et al. [34]' is that they were not clearly described (exercise prescriptions), making it difficult to apply them in clinical practice. And these problems can interfere with long-term results.

Therefore, this study will be the first clinical trial to compare the effects of adding power exercises to strength training with an isolated strength training program. Moreover, it will also analyze if these effects will be longer lasting than those of isolated strength training. The protocols will follow ACSM recommendations and will be described in detail to facilitate applications in clinical practice, and that could favor long-term benefits.

In relation to benefits, hip and knee strength training alone is able to improve pain and physical function in people with PFP [24-26], but only one study has evaluated long-term effects [21]. Adding power exercises to strength training can promote more consistent results in people with PFP. Furthermore, these results may be longer lasting than those of strength training alone (12 months).

Depending on the results of our study, we aim to change physiotherapist clinical practice by targeting hip and knee power exercises as a part of routine treatment for people with PFP. Usually, treatment for people with PFP includes hip and knee strength exercises and passive adjuncts (orthoses and insoles) and little or no attention to power exercises. In addition, because it follows the recommendations of the ACSM and exercises are described in detail (according to TIDieR), the protocol can be replicated in clinical practice and/or in scientific research.

\section{Trial status}

This trial is currently recruiting patients, the first patient was included on 12 August 2019, and this is the original version. To date, we have enrolled 37 study participants who have completed treatment, assessments, and follow- 
ups. We predict that recruitment will be completed in December 2021. Although existing data are being entered, no analysis has been performed yet.

\section{Supplementary Information}

The online version contains supplementary material available at https://doi. org/10.1186/s13063-021-05748-x.

Additional file 1: Supplementary Material.

\section{Acknowledgements}

The authors would like to specifically thank all the participants who will take part in the study. The authors would like to thank all the staff at the university.

\section{Ancillary and post-trial care}

After inclusion in the study, participants are given contact information on trial researchers and are asked to contact us if any trial-related health problem should arise. In these events, participants will be referred to the orthopedic doctor or to the necessary health service for the participant's prompt recovery.

\section{Dissemination policy}

The study results will be released in conferences, such as scientific conferences, internationally and nationally, and through articles published in peer-reviewed journals. This research is a part of a PhD thesis, and the publishing rights are owned by the authors. The results will be frequently presented to the supervising professor (FVS).

\section{Authors' contributions}

GSV, GSN, and FVS conceived the study. BCL and RFM performed the assessment. GSV, GKP, and MECBS were responsible for the interventions. GSV, GSN, and FVS were responsible for writing the paper. CJB critically reviewed the paper. All authors have read and approved the final manuscript and agreed to be accountable for all aspects of the work.

\section{Funding}

This trial has been conducted with no external funding (from funding agencies or the government); instead, the Graduate Program receives financial support in the form of scholarships, and the main researcher (GSV) receives a scholarship during her $\mathrm{PhD}$ period. This scholarship is part of the Coordination of Improvement of Higher Education Personnel - Brazil (CAPES) funding for Graduate Programs. However, there is no other direct funding from the Graduate Program for the development of the present study. CAPES did/will not have a role in the study design; collection, analysis, interpretation of data, and writing of the report.

\section{Availability of data and materials}

The dataset(s) supporting the conclusions of this article are available upon request from the authors.

\section{Declarations}

\section{Ethics approval and consent to participate}

The study was approved by the University Research Ethics Committee (CAAE: 12417019.8.0000.5504). Each participant will receive explanations regarding the study development. Participants will sign two copies of the Informed Consent Term (ICT); one copy will be for the volunteer and the other for the researcher. All collected data will be held confidential.

Any modification in study objectives, study design, study procedures, participant population, sample size, or significant administrative aspects will lead to a change in the study protocol. It will be under the supervision of the supervisor professor (FVS) and approved by the University Research Ethics Committee prior to implementing these amendments.

\section{Consent for publication}

Not applicable.

\section{Competing interests}

The authors declare that they have no financial and other competing interests.

\section{Author details}

'Physical Therapy Department, Federal University of Sao Carlos (UFSCar), Rodovia Washington Luis Km 235, São Carlos, São Paulo CEP 13565-905, Brazil. ${ }^{2}$ Physiotherapy and Rehabilitation Department, Federal University of Santa Maria (UFSM), Av Roraima 1000, Santa Maria, Rio Grande do Sul CEP 97105-900, Brazil. 'a Trobe Sport and Exercise Medicine Research Centre, School of Allied Health, La Trobe University, Melbourne, Australia. ${ }^{4}$ Department of Surgery, St Vincent's Hospital, University of Melbourne, Melbourne, Australia.

Received: 9 April 2021 Accepted: 25 October 2021

Published online: 06 November 2021

\section{References}

1. Davis IS, Powers CM. Patellofemoral pain syndrome: proximal, distal, and local factors - an international retreat. J Orthop Sports Phys Ther. 2010;40(3): A1-A48. https://doi.org/10.2519/jospt.2010.0302.

2. Smith BE, Selfe J, Thacker D, Hendrick P, Bateman M, Moffatt F, et al Incidence and prevalence of patellofemoral pain: a systematic review and meta-analysis. PLoS One. 2018;13(1):e0190892. https://doi.org/10.1371/journa I.pone.0190892.

3. Collins NJ, Bierma-Zeinstra SMA, Crossley KM, Van Linschoten RL, Vicenzino B, Van Middelkoop M. Prognostic factors for patellofemoral pain: a multicentre observational analysis. Br J Sports Med. 2013;47(4):227-33. https://doi.org/10.1136/bjsports-2012-091696.

4. Lankhorst NE, Van Middelkoop M, Crossley KM, Bierma-Zeinstra SMA, Oei EHG, Vicenzino B, et al. Factors that predict a poor outcome 5-8 years after the diagnosis of patellofemoral pain: a multicentre observational analysis. $\mathrm{Br} \mathrm{J}$ Sports Med. 2016;50(14):881-6. https://doi.org/10.1136/bjsports-2015-094664.

5. Rathleff MS, Rathleff CR, Olesen JL, Rasmussen S, Roos EM. Is knee pain during adolescence a self-limiting condition? Prognosis of Patellofemoral Pain and Other Types of Knee Pain. Am J Sports Med. 2016;44(5):1165-71. https://doi.org/10.1177/0363546515622456.

6. Crossley KM, Hinman RS. The patellofemoral joint: the forgotten joint in knee osteoarthritis. Osteoarthr Cartil. 2011;19(7):765-7. https://doi.org/10.101 6/j.joca.2011.05.005.

7. Hall R, Barber Foss K, Hewett TE, Myer GD. Sport specialization's association with na increased risk of developing anterior knee pain in adolescent female athletes. J Sport Rehabil. 2015;24(1):31-5. https://doi.org/10.1123/jsr.2 013-0101.

8. Prins MR, Van Der Wurff P. Females with patellofemoral pain syndrome have weak hip muscles: a systematic review. Aust J Physiother. 2009;55(1):9-15 https://doi.org/10.1016/S0004-9514(09)70055-8.

9. Rathleff MS, Rathleff CR, Crossley KM, Barton CJ. Is hip strength a risk factor for patellofemoral pain? A systematic review and meta-analysis. Br J Sports Med. 2014;48(14):1088. https://doi.org/10.1136/bjsports-2013-093305.

10. Duffey MJ, Martin DF, Cannon DW, Craven T, Messier SP. Etiologic factors associated with anterior knee pain in distance runners. Med Sci Sports Exerc. 2000;32(11):1825-32. https://doi.org/10.1097/00005768-20001100000003.

11. Kaya D, Citaker S, Kerimoglu U, Atay OA, Nyland J, Callaghan M, et al. Women with patellofemoral pain syndrome have quadriceps femoris volume and strength deficiency. Knee Surg Sports Traumatol Arthrosc. 2011; 19(2):242-7. https://doi.org/10.1007/s00167-010-1290-2.

12. Nunes GS, Barton CJ, Serrão FV. Hip rate of force development and strength are impaired in females with patellofemoral pain without signs of altered gluteus medius and maximus morphology. J Sci Med Sport. 2018;21(2):1238. https://doi.org/10.1016/j.jsams.2017.05.014.

13. Nunes GS, Barton CJ, Serrão FV. Impaired knee muscle capacity is correlated to impaired sagittal kinematics during jump landing in females with patellofemoral pain. J Strength Cond Res. 2020;Publish Ahead of Print. https://doi.org/10.1519/JSC.0000000000003616.

14. Nunes GS, De Oliveira SD, Crossley KM, Serrão FV, Pizzari T, Barton CJ. People with patellofemoral pain have impaired functional performance, that is correlated to hip muscle capacity. Phys Ther Sport. 2019a;40:85-90. https://doi.org/10.1016/j.ptsp.2019.08.010. 
15. Ferreira AS, de Oliveira SD. Barton CJ. Taborda B, Pazzinatto MF, et al. Impaired isometric, concentric, and eccentric rate of torque development at the hip and knee in patellofemoral pain. J Strength Cond Res: Briani RV; 2019.

16. Avraham F, Aviv S, Ya'akobi P, Faran H, Fisher Z, Goldman Y, et al. The efficacy of treatment of different intervention programs for patellofemoral pain syndrome-a single blinded randomized clinical trial. Pilot study. Sci World J. 2007;7:1256-62. https://doi.org/10.1100/tsw.2007.167.

17. Nakagawa TH, Muniz TB, Baldon RDM, Dias Maciel C, de Menezes Reiff RB, Serrão FV. The effect of additional strengthening of hip abductor and lateral rotator muscles in patellofemoral pain syndrome: a randomized controlled pilot study. Clin Rehabil. 2008;22(12):1051-60. https://doi.org/10.1177/02 69215508095357.

18. Fukuda TY, Rossetto FM, Magalhaes E, Bryk FF, Lucareli PR, De Almeida ACN. Short-term effects of hip abductors and lateral rotators strengthening in females with patellofemoral pain syndrome: a randomized controlled clinical trial. J Orthop Sports Phys Ther. 2010:40(11):736-42. https://doi.org/1 $0.2519 /$ jospt.2010.3246

19. Razeghi M, Etemadi Y, Taghizadeh S, Ghaem H. Could hip and knee muscle strengthening alter the pain intensity in patellofemoral pain syndrome? Iran Red Crescent Med J. 2010;12:104-10.

20. Dolak KL, Silkman C, Medina Mckeon J, Hosey RG, Lattermann C, Uhl TL. Hip strengthening prior to functional exercises reduces pain sooner than quadriceps strengthening in females with patellofemoral pain syndrome: a randomized clinical trial. J Orthop Sports Phys Ther. 2011;41(8):560-70. https://doi.org/10.2519/jospt.2011.3499.

21. Fukuda TY, Melo WP, Zaffalon BM, Rossetto FM, Magalhães E, Bryk FF, et al. Hip posterolateral musculature strengthening in sedentary women with patellofemoral pain syndrome: a randomized controlled clinical trial with 1year follow-up. J Orthop Sports Phys Ther. 2012;42(10):823-30. https://doi. org/10.2519/jospt.2012.4184.

22. Baldon R, Serrão FV, Scattone Silva R, Piva SR. Effects of functional stabilization training on pain, function, and lower extremity biomechanics in women with patellofemoral pain: a randomized clinical trial. J Orthop Sports Phys Ther. 2014:44(4):240-A8. https://doi.org/10.2519/jospt.2014.4940.

23. Ismail MM, Gamaleldein MH, Hassa KA. Closed kinetic chain exercises with or without additional hip strengthening exercises in management of patellofemoral pain syndrome: a randomized controlled trial. Eur J Phys Rehabil Med. 2013:49(5):687-98.

24. Lack S, Barton C, Sohan O, Crossley K, Morrissey D. Proximal muscle rehabilitation is effective for patellofemoral pain: a systematic review with meta-analysis. Br J Sports Med. 2015;49(21):1365-76. https://doi.org/10.1136/ bjsports-2015-094723.

25. Nascimento LR, Teixeira-Salmela LF, Souza RB, Resende RA. Hip and knee strengthening is more effective than knee strengthening alone for reducing pain and improving activity in individuals with patellofemoral pain: a systematic review with meta-analysis. J Orthop Sports Phys Ther. 2018;48(1): 19-31. https://doi.org/10.2519/jospt.2018.7365.

26. Collins NJ, Barton CJ, Van Middelkoop M, Callaghan MJ, Rathleff MS, Vicenzino BT, et al. Consensus statement on exercise therapy and physical interventions (orthoses, taping and manual therapy) to treat patellofemoral pain: recommendations from the 5th International Patellofemoral Pain Research Retreat, Gold Coast, Australia, 2017. Br J Sports Med, bjsports-2018099397

27. Domenech J, Sanchis-Alfonso V, López L, Espejo B. Influence of kinesiophobia and catastrophizing on pain and disability in anterior knee pain patients. Knee Surg Sport Traumatol Arthrosc. 2013;21(7):1562-8. https://doi.org/10.1007/s00167-012-2238-5.

28. Maclachlan LR, Collins NJ, Matthews MLG, Hodges MW, Vicenzino B. The psychological features of patellofemoral pain: a systematic review. $\mathrm{Br} J$ Sports Med. 2017;51(9):732-42. https://doi.org/10.1136/bjsports-2016096705.

29. Coburn SL, Barton CJ, Filbay SR, Hart HF, Rathleff MS, Crossley KM. Quality of life in individuals with patellofemoral pain: a systematic review including meta-analysis. Phys Ther Sport. 2018;33:96-108. https://doi.org/10.1016/j. ptsp.2018.06.006

30. Barton CJ, De Oliveira SD, Patterson BE, Crossley KM, Pizzari T, Nunes GS. A proximal progressive resistance training program targeting strength and power is feasible in people with patellofemoral pain. Phys Ther Sport. 2019; 38:59-65. https://doi.org/10.1016/j.ptsp.2019.04.010.
31. American College of Sports Medicine. American College of Sports Medicine position stand. Progression models in resistance training for healthy adults. Med Sci Sports Exerc. 2009;41(3):687-708. https://doi.org/10.1249/MSS.0b013 e3181915670.

32. Garber CE, Blissmer B, Deschenes MR, Franklin BA, Lamonte MJ, Lee IM, et al. American College of Sports Medicine position stand. Quantity and quality of exercise for developing and maintaining cardiorespiratory, musculoskeletal, and neuromotor fitness in apparently healthy adults: guidance for prescribing exercise. Med Sci Sports Exerc. 2011;43(7):1334-59. https://doi.org/10.1249/MSS.0b013e318213fefb.

33. Tyler TF, Nicholas SJ, Mullaney MJ, Mchugh MP. The role of hip muscle function in the treatment of patellofemoral pain syndrome. Am J Sports Med. 2006;34(4):630-6. https://doi.org/10.1177/0363546505281808.

34. Holden S, Rathleff MS, Jensen MB, Barton CJ. How can we implement exercise therapy for patellofemoral pain if we don't know what was prescribed? A systematic review. Br J Sports Med. 2017;52(6):385.

35. Eldridge SM, Chan CL, Campbell MJ, Bond CM, Hopewell S, Thabane L, et al. CONSORT 2010 statement: extension to randomised pilot and feasibility trials. BMJ. 2016;355:15239. https://doi.org/10.1136/bmj.i5239.

36. Hoffmann TC, Glasziou PP, Boutron I, Milne R, Perera R, Moher D, et al. Better reporting of interventions: template for intervention description and replication (TIDieR) checklist and guide. BMJ. 2014;348(mar07 3):g1687. https://doi.org/10.1136/bmj.g1687.

37. Chan A-W, Tetzlaff JM, Altman DG, Laupacis A, Gøtzsche PC, Krle A-Jerić K, et al. SPIRIT 2013 Statement: defining standard protocol items for clinical trials. Rev Panam Salud Publica. 2015:38(6):506-14.

38. Willy RW, Hoglund LT, Barton CJ, Bolgla LA, Scalzitti DA, Logerstedt DS, et al. Patellofemoral pain: clinical practice guidelines linked to the international classification of functioning, disability and health from the Academy of Orthopaedic Physical Therapy of the American Physical Therapy Association. J Orthop Sports Phys Ther. 2019;49(9):CPG1-CPG95. https://doi.org/10.2519/ jospt.2019.0302

39. Nakagawa TH, Moriya ÉTU, Maciel CD, Serrão FV. Frontal plane biomechanics in males and females with and without patellofemoral pain. Med Sci Sports Exerc. 2012;44(9):1747-55a.

40. Crossley KM, Bennell KL, Cowan SM, GreeN S. Analysis of outcome measures for persons with patellofemoral pain: which are reliable and valid? Arch Phys Med Rehabil. 2004;85(5):815-22. https://doi.org/10.1016/S0003-9993(03 00613-0.

41. Piva SR, Gil AB, Moore CG, Fitzgerald GK. Responsiveness of the activities of daily living scale of the knee outcome survey and numeric pain rating scale in patients with patellofemoral pain. J Rehabil Med. 2009;41(3):129-35. https://doi.org/10.2340/16501977-0295.

42. Mascal CL, Landel R, Powers C. Management of patellofemoral pain targeting hip, pelvis, and trunk muscle function: 2 case reports. J Orthop Sports Phys Ther. 2003;33(11):647-60. https://doi.org/10.2519/jospt.2 003.33.11.647.

43. Distefano LJ, Blackburn JT, Marshall SW, Padua DA. Gluteal muscle activation during common therapeutic exercises. J Orthop Sports Phys Ther. 2009. 39(7):532-40. https://doi.org/10.2519/jospt.2009.2796.

44. Reiman MP, Bolgla LA, Loudon JK. A literature review of studies evaluating gluteus maximus and gluteus medius activation during rehabilitation exercises. Physiother theory pract. 2012;28(4):257-68. https://doi.org/10.31 09/09593985.2011.604981.

45. Silva RS, Ferreira ALG, Nakagawa TH, Santos JE, Serrão FV. Rehabilitation of patellar tendinopathy using hip extensor strengthening and landingstrategy modification: case report with 6-month follow-up. J Orthop Sports Phys Ther. 2015;45(11):899-909. https://doi.org/10.2519/jospt.2015.6242.

46. Baechle TR, Earle RW, Wathan D. Resistance training. In: Essentials of Strength Training and Conditioning. Ed: Baechle, T. R., Earle, R. W 3rd edition. Champaign, IL: Human Kinetics, 2008.

47. Drinkwater EJ, Lawton TW, Lindsell RP, Pyne DB, Hunt PH, Mckenna MJ. Training leading to repetition failure enhances bench press strength gains in elite junior athletes. J Strength Cond Res. 2005;19(2):382-8. https://doi. org/10.1519/R-15224.1.

48. Izquierdo M, Ibanez J, Gonzalez-Badillo JJ, Hakkinen K, Ratamess NA, Kraemer WJ, et al. Differential effects of strength training leading to failure versus not to failure on hormonal responses, strength, and muscle power gains. J Appl Physiol. 2006;100(5):1647-56. https://doi.org/10.1152/ja pplphysiol.01400.2005. 
49. Robiner WN. Enhancing adherence in clinical research. Contemp Clin Trials. 2005;26(1):59-77. https://doi.org/10.1016/j.cct.2004.11.015.

50. Da Cunha RA, Costa LOP, Hespanhol Junior LC, Pires RS, Kujala UM, Lopes AD. Translation, cross-cultural adaptation, and clinimetric testing of instruments used to assess patients with patellofemoral pain syndrome in the Brazilian population. J Orthop Sports Phys Ther. 2013;43(5):332-9. https://doi.org/10.2519/jospt.2013.4228.

51. Kujala UM, Jaakkola LH, Koskinen SK, Taimela S, Hurme M, Nelimarkka O. Scoring of patellofemoral disorders. Arthroscopy. 1993;9(2):159e163.

52. Siqueira FB, Teixeira-Salmela LF, Magalhães LC. Análise das propriedades psicométricas da versão brasileira da escala tampa de cinesiofobia. Acta Ortopédica Bras. 2007;15(1):19-24. https://doi.org/10.1590/S1413-78522 007000100004.

53. Miller RP, Kori SH, Todd DD. The Tampa Scale. In: The Tampa Scale for Kinisophobia. Tampa, FL: Unpublished Report; 1991.

54. Goubert L, Crombez G, Van Damme S, Vlaeyen JW, Bijttebier P, Roelofs J. Confirmatory factor analysis of the Tampa Scale for Kinesiophobia: invariant two-factor model across low back pain patients and fibromyalgia patients. Clin J Pain. 2004;20(2):103-10. https://doi.org/10.1097/00002508-20040300000007.

55. Roos EM, Lohmander LS. The Knee injury and Osteoarthritis Outcome Score (KOOS): from joint injury to osteoarthritis. Health Qual Life Outcomes. 2003; 1(1):64. https://doi.org/10.1186/1477-7525-1-64

56. Roos EM, Roos HP, Lohmander LS, Ekdahl C, Beynnon BD. Knee Injury and Osteoarthritis Outcome Score (KOOS) — development of a self-administered outcome measure. J Orthop Sports Phys Ther. 1998;28(2):88-96. https://doi. org/10.2519/jospt.1998.28.2.88.

57. Souza RB, Powers CM. Differences in hip kinematics, muscle strength, and muscle activation between subjects with and without patellofemoral pain. $J$ Orthop Sports Phys Ther. 2009a;39(1):12-9. https://doi.org/10.2519/jospt.2 009.2885.

58. Baldon R, Nakagawa TH, Muniz TB, Amorim CF, Maciel CD, Serrao FV. Eccentric hip muscle function in females with and without patellofemoral pain syndrome. J Athl Train. 2009;44(5):490-6. https://doi.org/10.4085/10626050-44.5.490.

59. Bellew JW. A correlation analysis between rate of force development of the quadriceps and postural sway in healthy older adults. J Geriatr Phys Ther. 2002;25(1):11-5. https://doi.org/10.1519/00139143-200225010-00003.

60. Chang S-HJ, Mercer VS, Giuliani CA, Sloane PD. Relationship between hip abductor rate of force development and mediolateral stability in older adults. Arch Phys Med Rehabil. 2005;86(9):1843-50. https://doi.org/10.1016/ j.apmr.2005.03.006.

61. Suetta C, Aagaard P, Magnusson SP, Andersen LL, Sipilä S, Rosted A, et al. Muscle size, neuromuscular activation, and rapid force characteristics in elderly men and women: effects of unilateral long-term disuse due to hiposteoarthritis. J Appl Physiol. 2007;102(3):942-8. https://doi.org/10.1152/ja pplphysiol.00067.2006.

62. Andersen $L L$, Aagaard P. Influence of maximal muscle strength and intrinsic muscle contractile properties on contractile rate of force development. Eur 」 Appl Physiol. 2006;96(1):46-52. https://doi.org/10.1007/s00421-005-0070-z.

63. Jaeschke R, Singer J, Guyatt GH. Measurement of health status: ascertaining the minimal clinically important difference. Control Clin Trials. 1989;10(4): 407-15. https://doi.org/10.1016/0197-2456(89)90005-6.

64. Crowell MS, Wofford NH. Lumbopelvic manipulation in patients with patellofemoral pain syndrome. J Man Manip Ther. 2012; 20:113- 120. https:// doi.org/10.1179/204261861 2Y.0000000002.

65. Sterne JAC, White IR, Carlin JB, Spratt M, Royston P, Kenward MG, et al. Multiple imputation for missing data in epidemiological and clinical research: potential and pitfalls. BMJ. 2009;338(jun29 1):b2393. https://doi. org/10.1136/bmj.b2393.

66. Schafer JL, Graham JW. Missing data: our view of the state of the art. Psychol methods. 2002;7(2):147-77. https://doi.org/10.1037/1082-989X.7.2.147.

67. Crossley KM, Stefanik JJ, Selfe J, Collins NJ, Davis IS, Powers CM, et al. Patellofemoral pain consensus statement from the 4th International Patellofemoral Pain Research Retreat, Manchester. Part 1: Terminology, definitions, clinical examination, natural history, patellofemoral osteoarthritis and patient-reported outcome measures. Br J Sports Med. 2016a;50(14):83943. https://doi.org/10.1136/bjsports-2016-096384.

\section{Publisher's Note}

Springer Nature remains neutral with regard to jurisdictional claims in published maps and institutional affiliations.

\section{Ready to submit your research? Choose BMC and benefit from:}

- fast, convenient online submission

- thorough peer review by experienced researchers in your field

- rapid publication on acceptance

- support for research data, including large and complex data types

- gold Open Access which fosters wider collaboration and increased citations

- maximum visibility for your research: over $100 \mathrm{M}$ website views per year

At BMC, research is always in progress.

Learn more biomedcentral.com/submissions 